\title{
Comparison of Bayesian Estimation Methods for Rayleigh Progressive Censored Data Under the Different Asymmetric Loss Function
}

\author{
Reza Azimi ${ }^{1}$, Farhad Yaghmaei ${ }^{2}$ and Dariosh Azimi ${ }^{1}$ \\ ${ }^{1}$ Department of Statistics, Parsabad Moghan Branch \\ Islamic Azad University, Parsabad Moghan, Iran \\ Email: Azimireza1365@gmail.com,da_azimi@yahoo.com \\ ${ }^{2}$ Faculty of science, Golestan University, Gorgan, Golestan, Iran \\ Email: f_yaghmaei@yahoo.com
}

\begin{abstract}
In this paper, given a progressively type II censored sample from a Rayleigh distribution, the Bayesian estimators of parameter and Reliability function are obtained under asymmetric loss functions such as LINEX loss function, Precautionary loss function, entropy loss function for the parameter and reliability function. Comparisons of these estimators are made through simulation study.
\end{abstract}

Keywords: Bayesian estimation, Progressively censoring , Asymmetric loss function, Reliability function .

\section{Introduction}

The Rayleigh distribution provides a population model which is useful in several areas of statistics (for example Fernandez, 2000). In the literature, many researcher studied properties of the Rayleigh distribution, particularly in life testing and reliability. Life testing experiments often deal with censored sample in order to estimate the parameters involved in the life distribution in Bayesian literature. It is well known that, the performance of a Bayes estimator depends on the form of the prior distribution and the loss function. In view of this, given a progressively type II censored sample from a Rayleigh 
distribution, we obtain the Bayesian estimators under different asymmetric loss functions. The cumulative distribution function (cdf), and the reliability function of the Rayleigh distribution with parameter $\theta>0$ are

$$
\begin{array}{ll}
F(x \mid \theta)=1-\exp \left\{-\frac{\theta}{2} x^{2}\right\}, & x>0 \\
R(x \mid \theta)=\exp \left\{-\frac{\theta}{2} x^{2}\right\}, & x>0
\end{array}
$$

respectively.

Inferences for $\theta$ in the Rayleigh distribution have been discussed by several authors. Bayesian estimation and prediction problems for the $\theta$ based on doubly censored sample have been considered by Fernandez (2000) and Raqab and Madi (2002). Recently, Shuo et al. (2006) have derived the Bayesian estimator and prediction intervals based on progressively type II censored samples. A recent account on progressive censoring schemes can be obtained in the monograph by Balakrishnan and Aggarwala (2000) or in the excellent review article by Balakrishnan (2007).

Suppose that $n$ units are placed on a life test and the experimenter decides beforehand quantity $m$, the number of units, to be failed. Now at the time of the first failure, $R_{1}$ of the remaining $n-1$ surviving units are randomly removed from the experiment. Continuing on, at the time of the second failure, $R_{2}$ of the remaining $n-R_{1}-2$ units are randomly drawn from the experiment. Finally, at the time of the $m$ th failure, all the remaining $R_{m}=n-m-R_{1}-R_{2}-\ldots-R_{m-1}$ surviving units are removed from the experiment.

Suppose that $n$ independent items are put on a test and that the lifetime distribution of each item is given by the probability density function of (1). The ordered $m$-failures are observed under the type-II progressively censoring plan $\left(R, \ldots, R_{m}\right)$ where each $R_{i} \geq 0$ and $\sum_{j=1}^{m} R_{j}+m=n$. If the ordered $m$-failures are denoted by $x_{(1)}<x_{(2)}<\ldots<x_{(m)}$, then the likelihood function based on the observed sample $x_{(1)}<x_{(2)}<\ldots<x_{(m)}$ (for convenience notation are denoted by $\left.x_{1}<x_{2}<\ldots<x_{m}\right)$ is

$$
L(\theta)=c \prod_{i=1}^{m} f\left(x_{i} \mid \theta\right)\left[1-F\left(x_{i} \mid \theta\right)\right]^{R_{i}}
$$

where $c=n\left(n-1-R_{1}\right) \ldots\left(n-R_{1}-\ldots-R_{m-1}-m+1\right)$. According to (1) and (3), the latter function can be obtained as follows,

$$
L(\theta)=c \theta^{m} \exp \left\{-\frac{\theta}{2} \sum_{i=1}^{m}\left(R_{i}+1\right) x_{i}^{2}\right\} \prod_{i=1}^{m} x_{i} .
$$


It follows from (4) that logarithm of the latter function becomes proportional to

$$
l(\theta)=\log L(\theta) \propto m \log \theta-\frac{\theta}{2} \sum_{i=1}^{m}\left(R_{i}+1\right) x_{i}^{2} .
$$

The maximum likelihood estimator (MLE) of $\theta$, denoted by $\hat{\theta}$, can then be derived by solving the equation

$$
\frac{\partial l(\theta)}{\partial \theta}=\frac{m}{\theta}-\frac{1}{2} \sum_{i=1}^{m}\left(R_{i}+1\right) x_{i}^{2}=0
$$

Therefore MLE for $\theta$ can be obtained as the following form

$$
\hat{\theta}=\frac{2 m}{\sum_{i=1}^{m}\left(R_{i}+1\right) x_{i}^{2}}
$$

It is clear that the MLE of reliability function, $R(t \mid \hat{\theta})$, can be obtained by $\hat{R}(t \mid \hat{\theta})=\exp \left(-\frac{\hat{\theta}}{2} t^{2}\right)$.

\section{Bayesian estimation}

We now derive the Bayes estimator for the parameter $\theta$ based on the progressive Type-II censored data (for more detail see Shuo et al., 2006). Here we consider family of prior densities as the following form

$$
\pi(\theta)=a e^{-a \theta}
$$

By combining (4) and the latter prior density function, we can obtain posterior density of $\theta$ as the following form,

$$
\pi(\theta \mid \mathbf{X})=\frac{\left[a+\frac{1}{2} \sum_{i=1}^{m}\left(R_{i}+1\right) x_{i}^{2}\right]^{(m+1)}}{\Gamma(m+1)} \exp \left\{-\theta\left(a+\frac{1}{2} \sum_{i=1}^{m}\left(R_{i}+1\right) x_{i}^{2}\right)\right\} \theta^{m}
$$

where $\theta>0$. Substituting $\theta=(-2 \log s) / t^{2}$ into (6), we can obtain the posterior density function of $s=R(t \mid \theta)$ as

$$
\pi(s \mid \mathbf{X})=\frac{\left[a+\frac{1}{2} \sum_{i=1}^{m}\left(R_{i}+1\right) x_{i}^{2}\right]^{(m+1)}}{\Gamma(m+1)} s^{\frac{2}{t^{2}}\left(a+\frac{1}{2} \sum_{i=1}^{m}\left(R_{i}+1\right) x_{i}^{2}\right)-1}(-\log s)^{m}\left(\frac{2}{t^{2}}\right)^{(m+1)}
$$

where $0<s<1$. 


\subsection{Bayesian estimators under squared-error loss func- tion}

Under squared-error loss function, the Bayesian estimator of $\theta$ is the mean of the posterior density given by

$$
\hat{\theta}_{B}=E(\theta \mid \mathbf{X})=\frac{m+1}{a+\frac{1}{2} \sum_{i=1}^{m}\left(R_{i}+1\right) x_{i}^{2}}
$$

The Bayes estimator of $R(t \mid \theta)$ is given by

$$
\hat{R}_{t(B)}=E(R(T=t \mid \theta) \mid \mathbf{X})=\left(\frac{a+\frac{1}{2} \sum_{i=1}^{m}\left(R_{i}+1\right) x_{i}^{2}}{a+\frac{1}{2} \sum_{i=1}^{m}\left(R_{i}+1\right) x_{i}^{2}+t^{2} / 2}\right)^{m+1}
$$

and the posterior risk (minimum posterior expected loss) of $\hat{\theta}_{B}$ is equal to the posterior variance.

\subsection{Bayes estimators under Precautionary loss function}

Precautionary loss function which is asymmetric, was introduced by Norstrom (1996),

$$
L(\hat{\theta}, \theta)=\frac{(\hat{\theta}-\theta)^{2}}{\hat{\theta}} .
$$

The Bayes estimator under the latter loss function is denoted by $\hat{\theta_{p}}$ and may be obtained by solving the following equation

$$
{\hat{\theta_{p}}}^{2}=E\left(\theta^{2} \mid \mathbf{X}\right)
$$

Under Precautionary loss function, the Bayes estimator $\hat{\theta}_{p}$ of $\theta$ can be obtained as

$$
\hat{\theta_{p}}=\frac{\sqrt{(m+1)(m+2)}}{a+\frac{1}{2} \sum_{i=1}^{m}\left(R_{i}+1\right) x_{i}^{2}}
$$

And of $R(t \mid \theta)$ can be obtained as

$$
\hat{R}_{t(P)}=\sqrt{E\left((R(t \mid \theta))^{2} \mid \mathbf{X}\right)}=\sqrt{\left(\frac{a+\frac{1}{2} \sum_{i=1}^{m}\left(R_{i}+1\right) x_{i}^{2}}{a+\frac{1}{2} \sum_{i=1}^{m}\left(R_{i}+1\right) x_{i}^{2}+t^{2}}\right)^{m+1}}
$$

\subsection{Bayes estimators under entropy loss function}

In many practical situations, it appears to be more realistic to express the loss in terms of the ratio $\frac{\hat{\theta}}{\theta}$. In this case, Calabria and Pulcini (1994) point out that a useful asymmetric loss function is the entropy loss function,

$$
L(\delta) \propto\left[\delta^{P}-P \log _{e}(\delta)-1\right] .
$$


where

$$
\delta=\frac{\hat{\theta}}{\theta}
$$

whose minimum occurs at $\hat{\theta}=\theta$.the latter has been used in Dey et al., (1987) and Dey and Liu (1992), in the original form having $P=1$,

$$
L(\delta)=b\left[\delta-\log _{E}(\delta)-1\right], b>0 .
$$

Therefore, the Bayes estimator under the entropy loss function with $P=1$, is denoted by $\hat{\theta}_{E}$, is given by the following equation,

$$
\hat{\theta}_{E}=\left[E\left(\frac{1}{\theta} \mid \mathbf{X}\right)\right]^{-1} .
$$

It can be followed from the latter that,

$$
\begin{gathered}
\hat{\theta}_{E}=\frac{m}{\frac{1}{2} \sum_{i=1}^{m}\left(R_{i}+1\right) x_{i}^{2}+a} \\
\hat{R}_{t(E)}=\left[E\left(\frac{1}{R(t \mid \theta)}\right) \mid \mathbf{X}\right]^{-1}=\left(\frac{a+\frac{1}{2} \sum_{i=1}^{m}\left(R_{i}+1\right) x_{i}^{2}}{a+\frac{1}{2} \sum_{i=1}^{m}\left(R_{i}+1\right) x_{i}^{2}-t^{2} / 2}\right)^{-(m+1)}
\end{gathered}
$$

\subsection{Bayes estimators under LINEX loss function}

The LINEX loss function for $\theta$ can be expressed as the following proportional

$$
L(\Delta) \propto \exp (C \Delta)-C \Delta-1 ; C \neq 0
$$

where $\Delta=(\hat{\theta}-\theta)$ and $\hat{\theta}$ is an estimate of $\theta$. The Bayes estimator of $\theta$, denoted by $\hat{\theta_{L}}$ under the LINEX loss function is given by

$$
\hat{\theta_{L}}=-\frac{1}{C} \ln E_{\theta}[\exp (-C \theta)]
$$

It then follows from (6) and (14) we that,

$$
\begin{gathered}
\hat{\theta_{L}}=-\frac{(m+1)}{C} \ln \left(1-\frac{C}{\frac{1}{2} \sum_{i=1}^{m}\left(R_{i}+1\right) x_{i}^{2}+a+C}\right) \\
\hat{R}_{t(L)}=-\frac{1}{C} \ln E[\exp \{-C R(t \mid \theta)\}]
\end{gathered}
$$

In such a case for estimate under LINEX loss function we can expand $e^{-C s}$ also in taylor series, and approximate this estimator

$$
\hat{R}_{t(L)} \approx-\frac{1}{C} \ln \left(1-C\left(\frac{a(x)}{a(x)+t^{2} / 2}\right)^{m+1}+\frac{C^{2}}{2}\left(\frac{a(x)}{a(x)+t^{2}}\right)^{m+1}-\frac{C^{3}}{6}\left(\frac{a(x)}{a(x)+3 t^{2} / 2}\right)^{m+1}\right)
$$

where

$$
a(x)=a+\frac{1}{2} \sum_{i=1}^{m}\left(R_{i}+1\right) x_{i}^{2}
$$




\section{Simulation study}

Applying the algorithm of Balakrishnan and Aggarwala (2000), we used the following steps to generate a progressive Type II censored sample from the Rayleigh distribution.

(a) Simulate $m$ independent exponential random variables $Z_{1}, Z_{2}, \ldots, Z_{m}$.

This can be done using inverse transformation $Z_{i}=-\ln \left(1-U_{i}\right)$ where $U_{i}$ are independent uniform $(0,1)$ random variables.

(b)Set

$$
X_{i}=\frac{Z_{1}}{n}+\frac{Z_{2}}{n-R_{1}-1}+\frac{Z_{3}}{n-R_{1}-R_{2}-2}+\cdots+\frac{Z_{i}}{n-R_{1}-R_{2}-\cdots-R_{i-1}-i+1}
$$

for $i=1,2, \ldots, m$. This is the required progressively type-II censored sample from the standard exponential distribution.

(c) Finally, we set $Y_{i}=F^{-1}\left(1-\exp \left(-X_{i}\right)\right)$,for $i=1,2, \ldots, m$, where $F^{-1}($.$) is$ the inverse cumulative distribution function of the Rayleigh distribution.Then $Y_{1}, Y_{2}, \cdots, Y_{m}$ is the required progressively type-II censored sample from the distribution $F($.$) .$

(d)We compute the MLE of $\theta$ by (5).

(e)We compute the the Bayes estimates $\hat{\theta}_{B}, \hat{\theta}_{p}, \hat{\theta}_{E}, \hat{\theta_{L}}$ respectively, using (8), (10), (12), (15) and obtain different estimators for $R(t)$ by using (9), (11), (13), (16).

(f) We repeat the above steps 1000 times. We then obtain the means and the RMSEs (root mean squared error) for different censoring sizes $m$ and censoring schemes where

$$
R M S E=\sqrt{1000^{-1} \sum_{i=1}^{1000}\left(\phi-\hat{\phi}_{i}\right)^{2}}
$$

and $\hat{\phi}$ is the estimator of $\phi$.

In all above cases the prior parameters chosen as $a=1$ and $a=0.5$, which yield the generated value of $\theta=2$ as the true value. The true values of $R(t)$ in $t=0.5$ is obtained $R(0.5)=0.7788008$. The results are summarized in Tables 1-3.Table- 1 shows that when $\mathrm{a}=0.5$ (prior parameter) the Bayesian estimates under the Entropy loss function are doing better (have the smallest biased and RMSE) as compared with the estimates under the Precautionary loss function, LINEX loss function, Squared error loss function or MLE. In Table-2 we see Bayesian estimates under the Squared error loss function are doing better as compared with the estimates under Precautionary loss function, LINEX loss function, Entropy loss function or MLE, while the Bayesian approach under the LINEX loss function with $c=0.5$ has smallest RMSE. It is immediate to note that RMSE decreases as the sample size increases. On the other hand, for progressive censored sample, the Bayesian estimation methods under the 
LINEX loss function with $c=0.5$, Entropy and Squared error loss functions are underestimation, but MLE and Bayesian estimators under the Precautionary loss function and the LINEX loss function with $c=-0.5$ are overestimated.

\section{Conclusion}

TIn this paper, based on the progressively type II censored sample from a Rayleigh distribution, we consider the classical and Bayesian inference procedures to estimate the unknown parameter as well as the reliability function for Rayleigh distribution. Our interest in this stems from the fact that we anticipated the different estimators to be differently obtained under the symmetric (squared error, Shuo et al., 2006) and the asymmetric loss functions . In terms of our anticipation, we found that the Bayesian estimators based on progressively type II censoring are superior to the MLEs (simulation results). Differences between the different estimators arise when for censoring samples RMSEs decrease as sample size increases. For instance, according to Table-3, that the Bayesian estimators for Reliability function $R(t)$, under the Precautionary loss function have the smallest biased and smallest RMSE as compared with the estimates under the Entropy loss function, LINEX loss function, Squared error loss function or MLE. Then we suggest to use Bayesian approach under the Precautionary loss function in estimating the reliability function $R(t)$. Finally, although this study does not consider the problem of predicting for the future records, but the results of the simulation with considered sample size can be extended to this.

\section{References}

[1] Balakrishnan N.(2007). Progressive censoring methodology:an appraisal.(with discussions).TEST 16(2),211-296.

[2] Balakrishnan,N.,Aggarwala,R.(2000). Progressive Censoring: Theory, Methods, and Applications. Boston, MA: Birkhauser.

[3] Calabria, R. and Pulcini, G. (1994). An engineering approach to Bayes estimation for the Weibull distribution. Micro-electron. Reliab. 34 (5), 789-802.

[4] Dey, D.K. and Liu, Pei-San L.(1992). On comparison of estimators in a generalized life model, Micro-electron.Reliab. 32 (1), 207-221.

[5] Dey, D.K., Ghosh, M. and Srinivasan, C.(1987). Simultaneous estimation of parameters under entropy loss. J. Statist. Plan. and Infer., 347-363. 
[6] Fernandez, A. J.(2000). Bayesian inference from type II censored Rayleigh data. Statistics and Probability Letters, 48, 393-399.

[7] Shuo,J.W., Dar , H.C., Shyi ,T.C.(2006).Bayesian inference for Rayleigh distribution under progressive censored sample. Appl. Stoch. Models Bus. Ind. 22, 269-279.

[8] Raqab MZ, Madi MT.(2002). Bayesian prediction of the total time on test using doubly censored Rayleigh data. Journal of Statistical Computation and Simulation , 72, 781-789.

[9] Norstrom, J.G.(1996). The use of precautionary loss functions in risk analysis.IEEE Transactions on Reliability, 45 (3), 400-403.

[10] Zellner,A.(1986). Bayes estimation and prediction using asymmetric loss functions, Jour. Amer. Statist.Assoc. 81, 446-451.

Table 1: Averaged values of RMSEs for estimates of the parameter $\theta, \mathrm{a}=0.5$

\begin{tabular}{|c|c|c|c|c|c|c|c|c|}
\hline \multirow[t]{2}{*}{$n$} & \multirow[t]{2}{*}{$m$} & \multirow[t]{2}{*}{$\left(R_{1}, \ldots, R_{m}\right)$} & \multirow[t]{2}{*}{ MLE } & \multirow[t]{2}{*}{$\hat{\theta}_{B}$} & \multirow[t]{2}{*}{$\hat{\theta_{p}}$} & \multirow[t]{2}{*}{$\hat{\theta}_{E}$} & \multicolumn{2}{|c|}{$\hat{\theta}_{L}$} \\
\hline & & & & & & & $c=-0.5$ & $c=0.5$ \\
\hline \multirow{6}{*}{20} & 10 & $(5,5,0, \ldots, 0)$ & 2.235 & 2.188 & 2.286 & 1.989 & 2.318 & 2.078 \\
\hline & & & 0.8076 & 0.6855 & 0.7454 & 0.5991 & 0.8122 & 0.5963 \\
\hline & & $(4,2,2,2,0, . ., 0)$ & 2.206 & 2.161 & 2.258 & 1.965 & 2.288 & 2.053 \\
\hline & & & 0.8171 & 0.6886 & 0.7451 & 0.6094 & 0.8143 & 0.6016 \\
\hline & & $(0, \ldots, 0,2,2,2,4)$ & 2.213 & 2.170 & 2.266 & 1.972 & 2.297 & 2.062 \\
\hline & & & 0.7887 & 0.6704 & 0.7279 & 0.5901 & 0.7927 & 0.5850 \\
\hline \multirow[t]{6}{*}{30} & 15 & $(5,5,5,0, \ldots, 0)$ & 2.162 & 2.142 & 2.208 & 2.008 & 2.222 & 2.070 \\
\hline & & & 0.5972 & 0.5430 & 0.5790 & 0.4913 & 0.6087 & 0.4928 \\
\hline & & $(3,3,3,3,3,0, \ldots, 0)$ & 2.140 & 2.120 & 2.185 & 1.987 & 2.199 & 2.048 \\
\hline & & & 0.6428 & 0.5844 & 0.6180 & 0.5363 & 0.6498 & 0.5346 \\
\hline & & $(0, \ldots, 0,3,3,3,3,3)$ & 2.153 & 2.132 & 2.197 & 1.998 & 2.212 & 2.059 \\
\hline & & & 0.6435 & 0.5833 & 0.6181 & 0.5326 & 0.6508 & 0.5316 \\
\hline \multirow[t]{6}{*}{40} & 20 & $(5,5,5,5,0, \ldots, 0)$ & 2.106 & 2.095 & 2.144 & 1.995 & 2.152 & 2.042 \\
\hline & & & 0.5176 & 0.4844 & 0.5071 & 0.4523 & 0.5251 & 0.4523 \\
\hline & & $(2, \ldots, 2,0, \ldots, 0)$ & 2.109 & 2.098 & 2.148 & 1.999 & 2.155 & 2.046 \\
\hline & & & 0.4914 & 0.4606 & 0.4837 & 0.4284 & 0.5005 & 0.4293 \\
\hline & & $(0, \ldots, 0,2, \ldots, 2)$ & 2.097 & 2.086 & 2.135 & 1.987 & 2.143 & 2.033 \\
\hline & & & 0.5179 & 0.4849 & 0.5068 & 0.4546 & 0.5247 & 0.4538 \\
\hline
\end{tabular}


Table 2: Averaged values of RMSEs for estimates of the parameter $\theta, \mathrm{a}=1$

\begin{tabular}{|c|c|c|c|c|c|c|c|c|}
\hline \multirow[t]{2}{*}{$n$} & \multirow[t]{2}{*}{$m$} & \multirow[t]{2}{*}{$\left(R_{1}, \ldots, R_{m}\right)$} & \multirow[t]{2}{*}{ MLE } & \multirow[t]{2}{*}{$\hat{\theta}_{B}$} & \multirow[t]{2}{*}{$\hat{\theta_{p}}$} & \multirow[t]{2}{*}{$\hat{\theta}_{E}$} & \multicolumn{2}{|c|}{$\hat{\theta}_{L}$} \\
\hline & & & & & & & $c=-0.5$ & $c=0.5$ \\
\hline \multirow[t]{6}{*}{20} & 10 & $(5,5,0, \ldots, 0)$ & 2.181 & 1.941 & 2.027 & 1.764 & 2.039 & 1.855 \\
\hline & & & 0.7365 & 0.5032 & 0.5227 & 0.5117 & 0.5560 & 0.4777 \\
\hline & & $(4,2,2,2,0, . ., 0)$ & 2.212 & 1.956 & 2.043 & 1.778 & 2.057 & 1.868 \\
\hline & & & 0.8473 & 0.5471 & 0.5713 & 0.5429 & 0.6129 & 0.5114 \\
\hline & & $(0, \ldots, 0,2,2,2,4)$ & 2.218 & 1.965 & 2.052 & 1.786 & 2.066 & 1.877 \\
\hline & & & 0.7900 & 0.5224 & 0.5469 & 0.5197 & 0.5839 & 0.4897 \\
\hline \multirow[t]{6}{*}{30} & 15 & $(5,5,5,0, \ldots, 0)$ & 2.116 & 1.961 & 2.021 & 1.838 & 2.028 & 1.900 \\
\hline & & & 0.6144 & 0.4780 & 0.4916 & 0.4749 & 0.5124 & 0.4570 \\
\hline & & $(3,3,3,3,3,0, \ldots, 0)$ & 2.126 & 1.970 & 2.030 & 1.847 & 2.037 & 1.908 \\
\hline & & & 0.6030 & 0.4673 & 0.4816 & 0.4631 & 0.5020 & 0.4460 \\
\hline & & $(0, \ldots, 0,3,3,3,3,3)$ & 2.136 & 1.977 & 2.038 & 1.853 & 2.045 & 1.915 \\
\hline & & & 0.6414 & 0.4869 & 0.5028 & 0.4788 & 0.5260 & 0.4619 \\
\hline \multirow[t]{6}{*}{40} & 20 & $(5,5,5,5,0, \ldots, 0)$ & 2.119 & 2.0009 & 2.048 & 1.9056 & 2.052 & 1.952 \\
\hline & & & 0.5526 & 0.4489 & 0.4620 & 0.4378 & 0.4776 & 0.4287 \\
\hline & & $(2, \ldots, 2,0, \ldots, 0)$ & 2.100 & 1.986 & 2.033 & 1.891 & 2.037 & 1.938 \\
\hline & & & 0.5076 & 0.4199 & 0.4308 & 0.4141 & 0.4440 & 0.4039 \\
\hline & & $(0, \ldots, 0,2, \ldots, 2)$ & 2.104 & 1.989 & 2.036 & 1.894 & 2.040 & 1.941 \\
\hline & & & 0.5168 & 0.4279 & 0.4394 & 0.4208 & 0.4526 & 0.4113 \\
\hline
\end{tabular}


Table 3: Averaged values of RMSEs for estimates of the $R(t), \mathrm{a}=0.5$

\begin{tabular}{|c|c|c|c|c|c|c|c|c|}
\hline \multirow[t]{2}{*}{$n$} & \multirow[t]{2}{*}{$m$} & \multirow[t]{2}{*}{$\left(R_{1}, \ldots, R_{m}\right)$} & \multirow[t]{2}{*}{ MLE } & \multirow[t]{2}{*}{$\hat{R}_{t(B)}$} & \multirow[t]{2}{*}{$\hat{R}_{t(P)}$} & \multirow[t]{2}{*}{$\hat{R}_{t(E)}$} & \multicolumn{2}{|c|}{$\hat{R}_{t(L)}$} \\
\hline & & & & & & & $c=-0.1$ & $c=0.1$ \\
\hline \multirow[t]{6}{*}{20} & 10 & $(5,5,0, \ldots, 0)$ & 0.7619 & 0.7676 & 0.7701 & 0.7624 & 0.7125 & 0.8299 \\
\hline & & & 0.0682 & 0.0578 & 0.0561 & 0.0616 & 0.0825 & 0.0895 \\
\hline & & $(4,2,2,2,0, . ., 0)$ & 0.7580 & 0.7647 & 0.7673 & 0.7592 & 0.7099 & 0.8329 \\
\hline & & & 0.07710 & 0.0643 & 0.0623 & 0.0686 & 0.0878 & 0.0861 \\
\hline & & $(0, \ldots, 0,2,2,2,4)$ & 0.7524 & 0.7600 & 0.7627 & 0.7543 & 0.7058 & 0.8336 \\
\hline & & & 0.0825 & 0.0682 & 0.0660 & 0.0731 & 0.0926 & 0.0885 \\
\hline \multirow[t]{6}{*}{30} & 15 & $(5,5,5,0, \ldots, 0)$ & 0.7670 & 0.7703 & 0.7720 & 0.7668 & 0.7149 & 0.8364 \\
\hline & & & 0.0560 & 0.0504 & 0.0494 & 0.0526 & 0.0770 & 0.0809 \\
\hline & & $(3,3,3,3,3,0, \ldots, 0)$ & 0.7656 & 0.7690 & 0.7707 & 0.7654 & 0.7138 & 0.8324 \\
\hline & & & 0.0576 & 0.0516 & 0.0505 & 0.0539 & 0.0784 & 0.0798 \\
\hline & & $(0, \ldots, 0,3,3,3,3,3)$ & 0.7678 & 0.7711 & 0.7728 & 0.7676 & 0.7156 & 0.8357 \\
\hline & & & 0.0586 & 0.0524 & 0.0514 & 0.0547 & 0.0776 & 0.0821 \\
\hline \multirow[t]{6}{*}{40} & 20 & $(5,5,5,5,0, \ldots, 0)$ & 0.7667 & 0.7691 & 0.7704 & 0.7665 & 0.7139 & 0.8349 \\
\hline & & & 0.0496 & 0.0457 & 0.0450 & 0.0474 & 0.0755 & 0.0771 \\
\hline & & $(2, \ldots, 2,0, \ldots, 0)$ & 0.7714 & 0.7734 & 0.7747 & 0.7709 & 0.7177 & 0.8379 \\
\hline & & & 0.0439 & 0.0407 & 0.0401 & 0.0420 & 0.0703 & 0.0769 \\
\hline & & $(0, \ldots, 0,2, \ldots, 2)$ & 0.7691 & 0.7713 & 0.7726 & 0.7687 & 0.7177 & 0.8374 \\
\hline & & & 0.0474 & 0.0438 & 0.0432 & 0.0453 & 0.0731 & 0.0769 \\
\hline
\end{tabular}

